\title{
GP participation in increasing uptake in a national bowel cancer screening programme: the PEARL project
}

\author{
Sally C Benton ${ }^{*},{ }^{1,2}$, Piers Butler ${ }^{1}$, Katy Allen ${ }^{1}$, Michelle Chesters ${ }^{3}$, Sally Rickard ${ }^{4}$, Sally Stanley ${ }^{4}$, \\ Richard Roope ${ }^{4}$, Daniel Vulkan ${ }^{5}$ and Stephen W Duffy ${ }^{5}$ \\ ${ }^{1}$ NHS Bowel Cancer Screening Programme Southern Hub, Surrey Pathology Services, 20 Priestley Road, Surrey Research Park, \\ Guildford, Surrey, GU2 7YS, UK; ${ }^{2}$ Department of Biochemistry and Physiology, University of Surrey, Guildford, Surrey GU2 7XH, UK; \\ ${ }^{3}$ Wessex Cancer Clinical Network, Oakley Road, Southampton, Hampshire SO16 4GX, UK; ${ }^{4}$ The Whiteley Surgery, Yew Tree Drive, \\ Whiteley, Fareham PO15 7LB, UK and ${ }^{5}$ Centre for Cancer Prevention, Wolfson Institute of Preventive Medicine, Queen Mary \\ University of London, London EC1M 6BQ, UK
}

Background: The NHS Bowel Cancer Screening Programme (BCSP) in England does not involve general practitioners (GPs). Uptake is $\sim 58 \%$. The Practice Endorsed Additional Reminder Letter (PEARL) study piloted a GP-endorsed reminder letter.

\begin{abstract}
Methods: General practices in Wessex with uptake $<55 \%$ (prevalent invitations) were invited to participate. Subjects who had been invited for screening, sent a standard 28-day BCSP reminder letter but had not returned a test kit within 30 days of the standard reminder were sent a second reminder letter bearing the GP's letterhead and signature. Uptake was compared between PEARL and non-PEARL practices by standardised uptake ratio (standardised for prior prevalent uptake and other confounders). In addition, 25 non-PEARL practices were matched with PEARL practices for prior prevalent uptake and number of invitees.
\end{abstract}

Results: Twenty-five practices agreed to participate. A total of 3149 GP-endorsed reminders were sent. Uptake in the PEARL practices was $54 \%$ compared with $51 \%$ in the matched-control practices. The adjusted RR for uptake was 1.08 (95\% Cl: $1.05,1.11$, $P<0.001)$ for all invitees and $2.18(1.79,2.66, P<0.001)$ for invitees who had not returned a kit following the standard reminder.

Conclusions: The GP-endorsed reminder was associated with significantly increased uptake among subjects not responding to the standard reminder letter.

Worldwide, colorectal cancer (CRC) is a major health burden; in 2012, nearly 1.4 million cases were diagnosed and there were 694000 deaths (Ferlay et al, 2013). Screening using guaiac-based faecal occult blood testing (gFOBt) reduces mortality from CRC, as demonstrated by several population-based randomised controlled trials (Mandel et al, 1993; Kewenter et al, 1994; Hardcastle et al, 1996; Kronborg et al, 1996). The Bowel Cancer Screening Programme (BCSP) was implemented by the National Health Service (NHS) in England in 2006 and is delivered by five regional Hubs. Men and women aged 60-74 years (inclusive) receive an invitation to participate in screening using gFOBt every 2 years. The initial stage of the process is a pre-invitation letter that explains the disease and how the screening programme works. A week later, a gFOBt kit is sent out with another invitation letter. All correspondence from the Hub is signed by the BCSP Hub Director, who is most likely unknown to the subject receiving the letter due to the large geographical regions covered by each Hub.

The gFOBt requires participants to smear two small faecal samples from each of three separate stools onto a card that, when complete, is returned in the post to the BCSP Hub for analysis. Participants that receive a positive (abnormal) test result are referred for a follow-up investigation; if the test is negative (normal), participants will be invited to be screened again in 2 years' time until they reach the age of 75 (after which 
time individuals may self-refer). A reminder letter is sent to subjects where no action has occurred on the Bowel Cancer Screening System within 28 days of being sent a test kit. Screening episodes are closed if there is no response 17 weeks after the kit was sent.

In England, 'uptake' of screening is defined as the proportion of invited subjects that is adequately screened (definitive positive or definitive negative test result) within 26 weeks of invitation. Overall uptake in England was $58.2 \%$ in $2014 / 2015$ (personal communication, National Office), considerably lower than in the breast and cervical screening programmes (Douglas et al, 2016. There is wide variation in uptake across the country. BCSP data have demonstrated uptake to be as low as $35 \%$ in the most deprived areas and as high as 63\% in least deprived areas (von Wagner et al, 2011).

There is evidence to suggest that people are more likely to respond to an invitation to screening if they are encouraged to do so by their GP or another health professional (Cole et al, 2002; Senore et al, 2010; Zajac et al, 2010; Hewitson et al, 2011; Camilloni et al, 2013; Shankleman et al, 2014; Massat et al, 2014; Hall et al, 2015; Wardle et al, 2015).

The aim of the Practice Endorsed Additional Reminder Letter (PEARL) project was to develop and pilot a sustainable intervention to engage subjects in bowel cancer screening. The project was designed to test whether an additional reminder letter on GPheaded paper and with the signature of the subject's GP could encourage uptake. It is the result of collaboration between the BCSP Southern Hub, a group of GPs in Wessex (Hampshire, Dorset and the Isle of Wight) working for Macmillan Cancer Support and Cancer Research UK, in partnership with the Wessex Strategic Clinical Network.

\section{MATERIALS AND METHODS}

Target population. The BCSP Southern Hub serves the south of England (excluding London), sending 20000 invitations for screening every week. The PEARL project tested the intervention in the target area of Wessex. All general practices in Wessex with an uptake of less than 55\% ('prevalent' episodes only and calculated for all screening data up to 10 September 2013) were invited to participate. These practices were typically in areas of socioeconomic deprivation. The PEARL intervention targeted all subjects aged 60-74 years (inclusive), registered with a participating practice who were invited to take part in screening and had not responded to the standard 28-day BCSP reminder letter.

Recruitment and participants. Eligible practices were contacted by e-mail and regular mail to invite them to participate. If no reply was received, they were contacted by telephone. The deadline for agreement to participate was 31 May 2014. Participating practices agreed to receive an educational 1-h visit to learn about the BCSP, the PEARL project and to discuss the practice-specific statistical cancer profile-the National Cancer Intelligence Network general practice profile. Practices were offered a financial incentive (£250) for participating in the project.

Each practice signed a consent form and a confidentiality agreement and data exchange form, which set out the responsibilities of both the Hub and the GP practice (Supplementary web-Appendix A).

The text of a standard second reminder letter (Supplementary web-Appendix B) was approved by the BCSP National Office.

Each participating practice sent the Hub a paper or scanned electronic copy of the practice letterhead and a digital GP signature. One GP signed the letter for all patients from the practice. An electronic letter template was constructed for each practice.
Method. The PEARL intervention took place in Wessex between September 2014 and October 2015 and was designed to run for a fixed period of time. The flowchart in Figure 1 summarises the PEARL process. On the first Wednesday of each month, BCSP subject-level data (subject name, address, number of test kits sent, number of test kits returned, date of initial invitation, date standard 28-day reminder letter sent) for each participating practice were downloaded from the ORACLE-based BCSP database (Bowel Cancer Screening System). The full name, NHS number, and address of all non-responders whose kit had been sent out 60-90 days prior to the extract date were entered onto a spreadsheet for each practice and sent using secure mail. The broad 60-90-day range is due to the programme download being run on a single day each month, and using a 30-day moving window to ensure no subjects were missed. The practice reviewed each subject's history and indicated on the spreadsheet using a dropdown menu whether the subject should be sent an additional reminder.

GPs returned completed spreadsheets to the Hub by secure e-mail.

Ten working days after the initial list was sent, the list of subjects on returned GP spreadsheets was checked against a new download of subject-level data from Bowel Cancer Screening System to ensure that subjects remained non-respondent, were still registered with the same GP, and that there was no record of death. GP-endorsed reminder letters were printed for all eligible subjects with the appropriate GP letterhead and signature. This process was repeated 15 working days after the initial list for any GP spreadsheets returned late.

In any 1 month, if no non-responders were identified for a participating practice, the practice was notified by e-mail that they would not receive a list that month. Practices that did not return a completed spreadsheet were sent a reminder e-mail 5 working days after the spreadsheet was sent.

The average date of sending a GP-endorsed reminder was 99 days after initial invitation. Subjects in PEARL-registered practices not sent a reminder and in the non-PEARL practices were assigned an index date, the date when a PEARL reminder would have been sent if the practices were participating and the subject had not yet returned an adequate kit. Uptake, the primary outcome measure, was defined as the proportion of subjects (of the overall invited population and of those not adequately screened before the index date) that was adequately screened. Uptake was compared between PEARL practices and all other practices in the Southern Hub region with a prevalent uptake of less than 55\%. The comparison group included all routine screened Southern Hub subjects with an invitation on days that PEARL subjects were invited.

Subject variables were sex, age-group, and screening history (categorised as 'prevalent' first-time invitee or previous nonresponder or 'incident' previous responder, plus sequence of episode-first, second, third, etc). In addition, a measure of social deprivation (index of multiple deprivation (IMD)) was assigned for each subject with reference to the English indices of deprivation. The IMD scores were grouped into quintiles based on national distributions with the use of predefined national cutoffs.

Statistical methods. Both the 25 PEARL-registered practices and the 1575 comparison practices were specified to have prevalent screen participation rates of less than $55 \%$ prior to the intervention. However, even within this range, the comparison practices had substantially higher prior participation rates than the PEARLregistered practices (see Results section). We therefore analysed the data analogously to standardised incidence or mortality ratios. Using logistic regression, in subjects within the comparison practices only, we estimated the effects of age, sex, prior prevalent participation, IMD quintile, episode type (prevalent or incident), and episode sequence on the probability of participation. These 


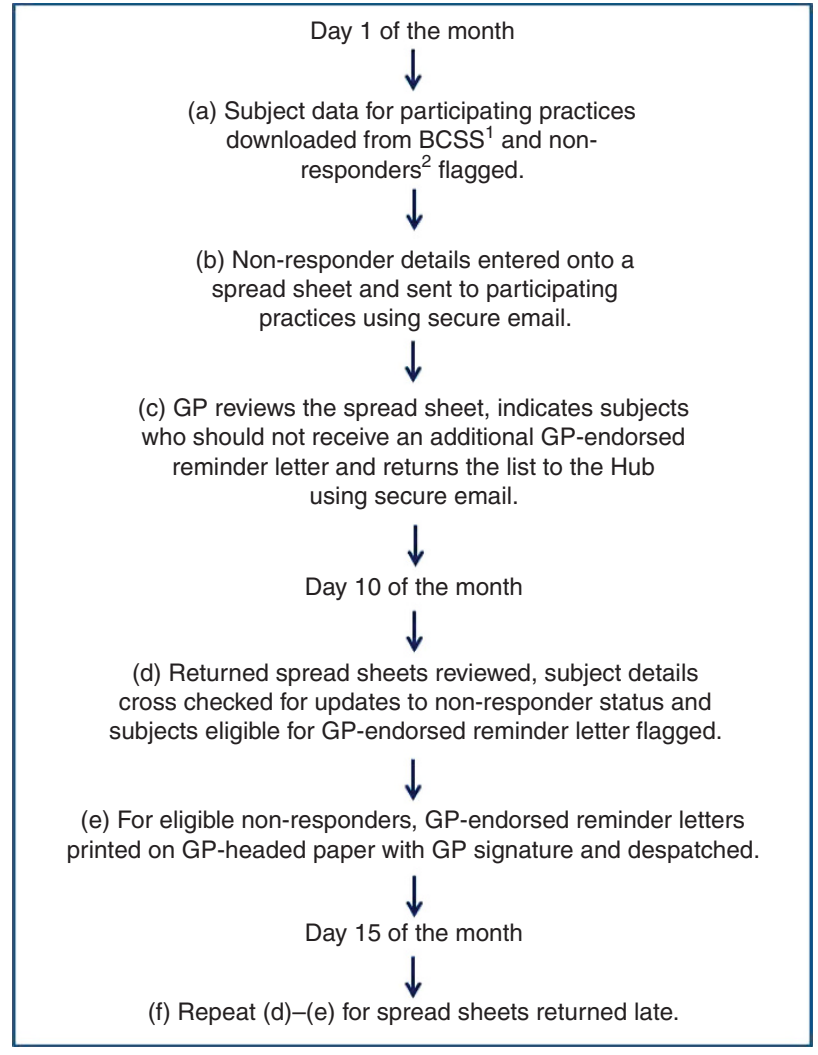

Figure 1. Schematic representation of PEARL process. ${ }^{1}$ BCSS: Bowel Cancer Screening System; ${ }^{2}$ Non-responders defined as subjects who had not returned a test kit within 30-60 days of a standard reminder letter.

were applied to the subjects in the PEARL-registered practices to calculate the expected proportion of participants in these practices (see Supplementary web-Appendix C for mathematical details). The effect of the intervention was estimated as the observed number of participants in the PEARL-registered practices divided by the expected number. We calculated confidence intervals on the ratio assuming a binomial distribution of numbers participating, taking into account the uncertainty in the expected numbers.

For subjects in the non-PEARL-registered practices, the index date was defined as the date on which a PEARL reminder would have been due if these had been PEARL subjects. Our primary analysis was to compare the participation rate of subjects in the PEARL-registered practices with that expected from the comparison practices, for overall participation and for participation in those not returning an adequate kit by the index date, unstratified by demographic or screening data. Secondary analysis consisted of the same comparisons in subgroups of age, sex, screening episode type (prevalent or incident), and IMD quintile.

As a check on our method, we also selected 25 non-PEARL practices, matched to the PEARL-registered practices by previous prevalent participation rates and number of invitees. We repeated our primary analysis, directly comparing participation rates in PEARL-registered practices with those in the matched non-PEARL practices. Analysis for this comparison was performed using inverse variance weighted average estimation conditioning on matched set (Cummings, 2009). All analyses were performed in Stata version 13.1 (StataCorp, 2013).

Funding and ethics. The PEARL project protocol was approved by the NHS BCSP Research Committee in March 2015 (reference ID 148). The Office for Data Release granted permission for sharing BCSP data with Professor Stephen Duffy (statistician) in November 2015 (reference ODR1516_154). Patient consent was not required under Section 251 of the 2006 NHS Act. The PEARL project is part of the ACE (Accelerate, Coordinate, Evaluate) programme, NHS England's initiative supported by Cancer Research UK and Macmillan Cancer Support to diagnose cancer early and improve outcomes. Funding was received from the National Cancer Intelligence Network.

\section{RESULTS}

Of the 43 eligible practices approached, 25 agreed to participate in the PEARL study. Over the 14-month study period, 324 nonparticipating subject lists were sent to GP practices. Seventeen practices had lists to review every month. For 11 of the study months, there were practices $(n=1-7)$ that received no list. The mean number of subjects on each list was 16.02 (range 1-103).One hundred twenty-four (38.3\%) lists were not returned. Two of the practices that signed up to the PEARL intervention did not return any of the lists.

One hundred sixty-one $(5.11 \%)$ subjects were deemed unsuitable by the GP to receive a reminder letter for the following reason:

- Patients receiving end-of-life care $(n=10)$

- Already having regular colonoscopy surveillance $(n=20)$

- Recent ( $<12$ months) diagnosis of bowel cancer $(n=4)$

- Unable to have colonoscopy $(n=5)$

- Other $(n=122)$.

There were 12878 invitees in the PEARL-registered practices and 1248689 in the non-PEARL-registered practices. A total of 3149 GP-endorsed reminder letters were sent.

Table 1 shows the characteristics of subjects in the PEARLregistered practices, in those practices matched to the PEARL 
practices, and in all the comparison practices. There were substantial differences between the invitees in the PEARLregistered practices and those in the non-PEARL-registered practices. In particular, the former were much more likely to be in deprived IMD categories. This is reflected in the fact that average prior prevalent participation in the PEARL-registered practices was $36 \%$, substantially lower than that observed in the non-PEARL practices, $47 \%$. This necessitated the approach described above, deriving results standardised for age, sex, IMD quintile, episode type, episode sequence, and prior prevalent participation. The differences between the PEARL practices and the matched practices are much attenuated, and the average prior prevalent participation in the matched practices was $36 \%$, as in the PEARL-registered practices. Table 2 shows the numbers returning a completed kit by the index date in the same groups.

Table 3 shows the basic results with respect to participation. There was a highly significant $(P<0.001)$ difference between the participation rates in the PEARL practices and that expected from the non-PEARL, with 6914 (54\%) participation vs 6543 (51\%) expected. In those who qualified for a reminder, that is, those who had not returned a completed kit by the index date, there was also a highly significant difference $(P<0.001)$ with $362(7 \%)$ participation in the PEARL practices compared with 167 (3\%) expected. The direct comparison of the 25 PEARL practices with the 25 matched practices showed slightly stronger but essentially similar results (Table 4 ).

Table 5 shows the subgroup results by age, sex, screen type (prevalent/incident), and socioeconomic status as measured by
IMD quintile, for all invitees, and for those not returning a completed kit by the index date. In the upper half of the table, all invitees, it can be seen that significantly greater participation than expected was noted in the PEARL practices for all subgroups except for subjects aged 65 years or less, with absolute differences ranging from 2 to $5 \%$. The difference in the effect of the PEARL intervention between age groups was statistically significant $(P=0.001)$. No other significant heterogeneity of the PEARL intervention effect between subgroups was observed.

In the corresponding results in the lower half of the table, for those with no return of an adequate kit before the index date, all subgroups showed highly significant results, with an approximate doubling of the rate of participation, typically from $2-3$ to $6-8 \%$. Again, there was significant heterogeneity of the effect by age, with a stronger effect of the PEARL intervention in those older than 65 years. No other significant heterogeneity of the intervention effect was observed between subgroups.

Using the method and rates given by Raine et al (2016), we predict the effect of increased uptake of screening from PEARL on observed outcomes nationally. In the 2014/2015 fiscal year, 4117866 people were invited for screening by the BCSP in England. An increase of $3 \%$ suggests that if PEARL was implemented nationally then an extra 123536 each year would be screened. In 2014/2015, the positivity rate among the screened population was $1.79 \%$, and $87.39 \%$ of these attend a specialist screening practitioner clinic. In all, $8.09 \%$ will have a colorectal cancer and $23.43 \%$ will have medium- or high-risk polyps. Hence, if PEARL were implemented nationally, this could detect up

Table 1. Characteristics of invitees in PEARL and comparison practices

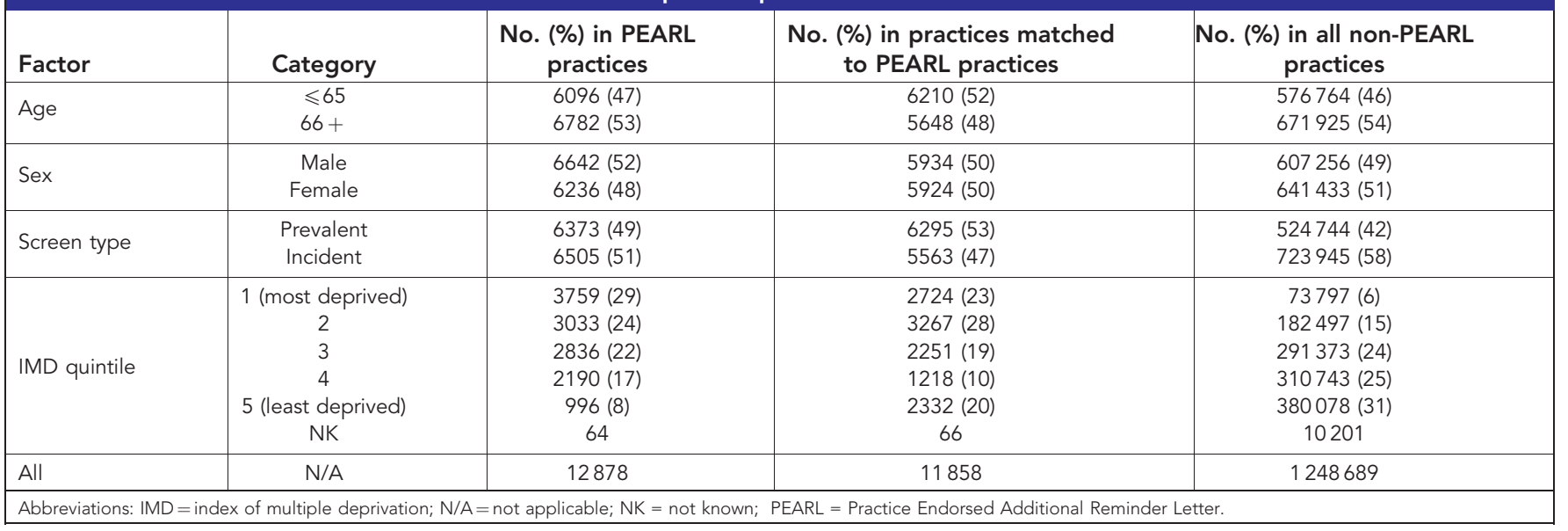

Table 2. Numbers (\%) adequately screened on or before the index date

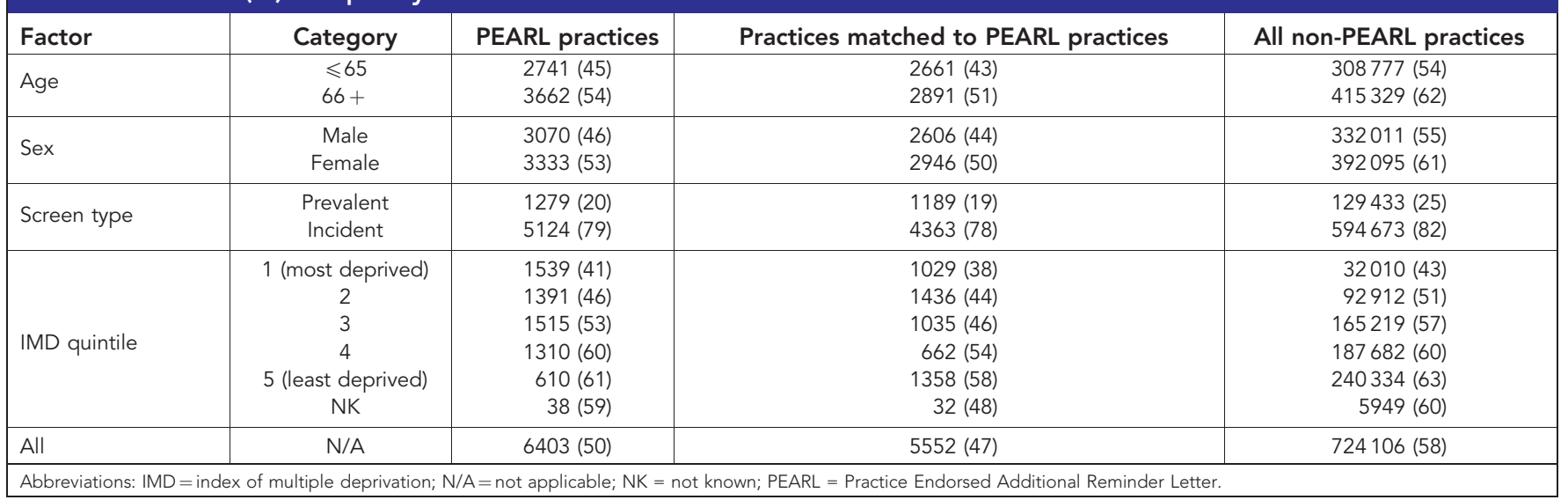


Table 3. Effect of intervention on participation in PEARL practices

\begin{tabular}{|l|c|c|c|c|}
\hline Population & $\begin{array}{c}\text { Observed/expected participating } \\
\text { subjects in PEARL practices }\end{array}$ & No. (\%) participating & O/E (95\% Cl) & Significance \\
\hline All invitees & $\begin{array}{l}\text { Observed } \\
\text { Expected }\end{array}$ & $\begin{array}{l}6914(54) \\
6543(51)\end{array}$ & $1.06(1.03-1.09)$ & $P<0.001$ \\
\hline $\begin{array}{l}\text { Those not returning on } \\
\text { or before index date }\end{array}$ & $\begin{array}{l}\text { Observed } \\
\text { Expected }\end{array}$ & $\begin{array}{l}362(7) \\
167(3)\end{array}$ & $2.17(1.96-2.40)$ & $P<0.001$ \\
\hline \multicolumn{2}{|l|}{ Abbreviations: $\mathrm{Cl}=$ confidence interval; O/E = Observed/Expected; PEARL = Practice Endorsed Additional Reminder Letter. } \\
\hline
\end{tabular}

\section{Table 4. Effect of intervention on participation from the direct comparison with matched practices}

\begin{tabular}{|c|c|c|c|c|}
\hline Population & Study group & No. (\%) participating & $\operatorname{RR}(95 \% \mathrm{Cl})$ & Significance \\
\hline All invitees & $\begin{array}{c}\text { Comparison practices } \\
\text { PEARL practices }\end{array}$ & $\begin{array}{l}5861 / 11858(49) \\
6914 / 12878(54)\end{array}$ & $\begin{array}{l}1.00(-) \\
1.08(1.05-1.11)\end{array}$ & $P<0.001$ \\
\hline Those not returning on or before index date & $\begin{array}{c}\text { Comparison practices } \\
\text { PEARL practices }\end{array}$ & $\begin{array}{l}144 / 5417(3) \\
362 / 5535(7)\end{array}$ & $\begin{array}{l}1.00(-) \\
2.18(1.79-2.66)\end{array}$ & $P<0.001$ \\
\hline
\end{tabular}

Table 5. Effect of intervention on participation in subgroups of PEARL practices by age, sex, screen type, and IMD quintile

\begin{tabular}{|c|c|c|c|c|c|c|}
\hline \multirow[b]{2}{*}{ Population } & \multirow[b]{2}{*}{$\begin{array}{l}\text { Demographic } \\
\text { subgroup }\end{array}$} & \multicolumn{2}{|c|}{$\begin{array}{l}\text { Observed and expected numbers } \\
\text { adequately screened, and total } \\
\text { subjects }\end{array}$} & & \multicolumn{2}{|c|}{ Significance } \\
\hline & & Observed (\%) & Expected (\%) & $\begin{array}{c}\text { O/E }(95 \% \mathrm{Cl}) \\
\text { Total }\end{array}$ & & \\
\hline All subjects & $\begin{array}{l}\text { Age } \leqslant 65 \text { years } \\
\text { Age }>65 \text { years } \\
\text { Males } \\
\text { Females } \\
\text { Prevalent screen } \\
\text { Incident screen } \\
\text { Most deprived quintile } \\
\text { Other four quintiles }\end{array}$ & $\begin{array}{l}2987(49) \\
3927(58) \\
3347(50) \\
3567(57) \\
1465(23) \\
5449(84) \\
1657(44) \\
5218(58)\end{array}$ & $\begin{array}{l}2966(49) \\
3577(53) \\
3184(48) \\
3359(54) \\
1345(21) \\
5197(80) \\
1578(42) \\
4933(54)\end{array}$ & $\begin{array}{l}6096 \\
6782 \\
6642 \\
6236 \\
6373 \\
6505 \\
3759 \\
9055\end{array}$ & $\begin{array}{l}1.01(0.97-1.05) \\
1.10(1.06-1.14) \\
1.05(1.01-1.09) \\
1.06(1.02-1.10) \\
1.09(1.03-1.15) \\
1.05(1.02-1.08) \\
1.05(1.00-1.11) \\
1.06(1.03-1.09)\end{array}$ & $\begin{array}{c}P=0.6 \\
P<0.001 \\
P=0.005 \\
P<0.001 \\
P=0.001 \\
P<0.001 \\
P=0.05 \\
P<0.001\end{array}$ \\
\hline $\begin{array}{l}\text { Subjects not returning an } \\
\text { adequate kit by the index date }\end{array}$ & $\begin{array}{l}\text { Age } \leqslant 65 \text { years } \\
\text { Age }>65 \text { years } \\
\text { Males } \\
\text { Females } \\
\text { Prevalent screen } \\
\text { Incident screen } \\
\text { Most deprived quintile } \\
\text { Other four quintiles }\end{array}$ & $\begin{array}{l}181(6) \\
181(7) \\
195(6) \\
167(7) \\
157(4) \\
205(20) \\
87(5) \\
274(8)\end{array}$ & $\begin{array}{r}104(4) \\
63(2) \\
95(3) \\
73(3) \\
70(2) \\
97(9) \\
45(2) \\
122(3)\end{array}$ & $\begin{array}{l}2949 \\
2586 \\
3058 \\
2477 \\
4484 \\
1051 \\
1919 \\
3593\end{array}$ & $\begin{array}{l}1.74(1.49-2.03) \\
2.87(2.42-3.41) \\
2.05(1.78-2.36) \\
2.29(1.96-2.67) \\
2.24(1.90-2.63) \\
2.11(1.83-2.43) \\
1.93(1.56-2.39) \\
2.25(1.99-2.54)\end{array}$ & $\begin{array}{l}P<0.001 \\
P<0.001 \\
P<0.001 \\
P<0.001 \\
P<0.001 \\
P<0.001 \\
P<0.001 \\
P<0.001\end{array}$ \\
\hline
\end{tabular}

to an additional 453 people $\left(123536^{\star} 0.0179^{\star} 0.8739^{\star} 0.2343\right)$ with high- or intermediate-risk polyps, and 156 people $\left(123536^{\star} 0.0179^{\star} 0.8739^{\star} 0.0809\right)$ with a colorectal cancer each year.

\section{DISCUSSION}

Our results indicate that the addition of a GP-endorsed reminder at 3 months significantly increased participation in the NHS BCSP, by $3 \%$ in absolute terms ( $54 \%$ vs $51 \%$ ), in general practices with participation rates below the national average. There is already evidence that primary care endorsement improves participation in screening for a number of cancers (Duffy et al, 2016) and this study provides further evidence to support this.

Our aim was to develop a robust, feasible, and sustainable method to increase uptake through direct GP endorsement of the BCSP in England. Although previous studies have demonstrated that GP endorsement is successful in increasing uptake in the programme (Cole et al, 2002; Senore et al, 2010; Zajac et al, 2010; Hewitson et al, 2011; Camilloni et al, 2013), these studies have not considered the long-term feasibility of uptake initiatives directly involving GP practices and, to our knowledge, none has targeted non-responders and asked GPs to assess the appropriateness of sending a subject a reminder letter.

Hall et al (2015) conducted a qualitative study using in-depth interviews with BCSP non-participants and found that nonparticipation in screening was not necessarily associated with negative attitudes towards screening or a decision not to return a kit. The authors concluded that some non-participants may have a degree of intention to take part in screening in the future and may be more responsive to interventions based on professional endorsement and reminders. The results from our study support this.

Another RCT was conducted in the south of England in 2009 among 20 general practices (1288 subjects invited for screening) (Hewitson et al, 2011). Subjects were randomised to either a GPendorsed letter and/or an enhanced information leaflet with their gFOBT kit. The GP-endorsed letter and the enhanced procedural leaflet increased uptake by $5.8 \%$ and $6.0 \%$, respectively, and had an additive effect (11.8\%). 
Although we have made the assumption that it is the GP endorsement that has led to our observed increase in uptake, due to the design of our study we cannot be certain whether an additional reminder letter from the Hub would in itself increase uptake in the programme. As summarised by Duffy et al (2016), reminders have been demonstrated to increase uptake in screening programmes. However, only two studies have reported the effect of postal reminders alone on participation, one of pre-appointment breast screening reminders (Allgood et al, 2016) and one of reminders following nonattendance in cervical screening (Eaker et al, 2004). Although both studies found an increase in participation with the reminders, it is not clear whether these results would generalise to the Bowel Cancer Screening Programme in the UK. To our knowledge, no studies assessing the impact of a second reminder letter have been published. In our primary standardised analysis, the effect of the intervention in terms of raw numbers was to increase participation by 371 (69146543) when all invitees are considered. Restricting analysis to those who had not returned an adequate kit by the index date (the target population of the intervention), the effect was an increase of 195 (362167). In the matched analysis, the figures would be 512 (6914-6914/ 1.08) (Table 4) and 196 (362-362/2.18), respectively. One could therefore be confident that the effect of adopting these reminders as policy would lie within the range of these figures. In terms of the larger number of participants estimated to accrue when all invitees are considered, it is not out of the question that the intervention had some effect on participation among those not sent a PEARL reminder but living with a subject who did receive a reminder.

During the intervention period, 3149 GP-endorsed reminders were sent in the PEARL-registered practices. The resulting absolute increase in participation was of the order of 200 in the target population. This is consistent with the observation that of those adequately screened, but not before the index date, in the PEARLregistered practices, 250 actually received a reminder. For each extra participant, between 6 and 16 PEARL reminders had to be sent, depending on which estimate one considers. The PEARLregistered practices were characterised by low socioeconomic status (Table 1), therefore the intervention has potential to improve delivery to traditionally underserved populations.

A limitation of this study is that the intervention was not randomised. However, we did include in the PEARL practices those which registered whether or not they supplied lists, to approximate an intention to treat analysis. Also, the similar results by two separate approaches, taking account of differences between the PEARL practices and the comparison practices, give some confidence in the results.

Two practices that had agreed to take part in the PEARL project did not return any non-responder patient lists but the practices may still have used the information to target patients-it is not possible to quantify that effect. The exclusion of non-responders by GPs could not be replicated in the control population, but this limitation would be likely to diminish the observed effect of the intervention. The process is dependent on engagement and active participation of GP practices and our experience demonstrated that logistical factors such as use of a generic e-mail address, key staff members leaving can impact the success of such projects.

The Hub process to generate the GP-endorsed second reminder letter was robust and became incorporated into the routine workload of the Southern Hub. For the majority of subjects, the fact the letter was signed by the subject's own GP practice and appeared on the GP's letterhead (as opposed to a letter signed by an unknown Hub Director) is likely to have had a positive effect on the subject's attitude. Other research has demonstrated that uptake of screening can be improved by direct contact in person with a GP or other health professional (Senore et al, 2010), especially in ethnically diverse populations (Shankleman et al, 2014; Massat et al, 2014), although such an approach has important resource implications in terms of cost and time and is probably unsustainable.
The results of this project require qualification, not least the labour-intensive method and high level of quality control required. Despite offering a financial incentive, in addition to the potential benefit to their patients, only $58 \%$ of invited GP practices agreed to participate. Anecdotal reasons given by practices for not participating in the PEARL project included the amount of time that would have to be invested, an unusually high turnover of practice managers, and organisational disruption caused by practice mergers during the study period.

The reasons given above for non-participation should be interpreted with caution, The practices invited to take part were those with the lowest uptake ( $<55 \%$ for prevalent invitations) and the link between deprivation and low uptake of bowel cancer screening has been firmly established. It may be that the observations should not be extrapolated to all GP surgeries on a national scale.

A key consideration for the study was the GP-patient relationship if a letter was coming on GP letter headed paper. This was the reason the GP was asked to review a list of their patients who had not responded and who were on the list to receive a reminder. It is notable that a reasonable number $(5.11 \%)$ of subjects were deemed unsuitable to receive the letter from the GP suggesting that sending out GP-personalised letters without having a GP review in advance might be inappropriate.

As part of the ASCEND randomised controlled trial in England (a national trial designed to reduce the social gradient in uptake), investigators sought permission from general practices to allow the practice name to be added to the standard BCSP pre-invitation letter (Wardle et al, 2015; Raine et al, 2016). In all, $80 \%$ of practices agreed. An increase in uptake of 0.7 percentage points was reported (58.2\% vs 57.5\%). The authors concluded that, given the willingness shown by GPs to endorse the BCSP, the small oneoff cost incurred to modify the standard invitation letter and the overall increase in uptake, the BCSP should consider adding the GP endorsement to the screening invitation letter. The London BCSP Hub have implemented the GP endorsement.

Compared with the ASCEND GP-endorsed pre-invitation intervention (Raine et al, 2016), the PEARL project has demonstrated a greater impact on uptake, possibly because subjects received a second reminder that appeared to have been sent by their own GP. Consideration does need to be given to the feasibility of PEARL compared with ASCEND. The ASCEND approach requires only that a GP practice agrees once a year for the endorsement to be added to the standard letters. In contrast, PEARL required continuous engagement from GP practices and created additional work for the Hub. The benefits of the PEARL approach are clear by the increase in uptake, whether this is sustainable across the country is questionable when considering the pressures that GPs face.

We have developed a robust and sustainable method to send GPendorsed letters to non-responders. A GP-endorsed second reminder letter significantly increased uptake, by about 3 percentage points, both as a proportion of all invitees or only those who had not returned a test kit by the index date. The extra work required for the Hub and GPs to support the PEARL intervention should be evaluated and recommendations made on the feasibility of rolling out this process nationally within BCSP. The process would require refinement before being rolled out on a larger scale.

\section{ACKNOWLEDGEMENTS}

The authors acknowledge the contribution made by Professor Stephen Halloran who, along with Richard Roope, conceived the idea for this study. Stephen Duffy participated in this work as part of the ACE programme within the responsive programme of the Policy Research Unit (PRU) in Cancer Awareness, Screening and Early 
Diagnosis. The PRU receives funding for a research programme from the Department of Health Policy Research Programme. It is a collaboration between researchers from seven institutions (Queen Mary University of London, UCL, King's College London, London School of Hygiene and Tropical Medicine, Hull York Medical School, Durham University, and Peninsula Medical School).

\section{CONFLICT OF INTEREST}

The authors declare no conflict of interest.

\section{REFERENCES}

Allgood PC, Maxwell AJ, Hudson S, Offman J, Hutchison G, Beattie C, Tuano-Donnelly R, Threlfall A, Summersgill T, Bellis L, Robinson C, Heaton S, Patnick J, Duffy SW (2016) A randomised trial of the effect of postal reminders on attendance for breast screening. Br J Cancer 114: 171-176.

Camilloni L, Ferroni E, Cendales BJ, Pezzarossi A, Furnari G, Borgia P, Guasticchi G, Giorgi Rossi P (2013) Methods to increase participation in organised screening programs: a systematic review. BMC Public Health 13: 464.

Cole SR, Young GP, Byrne D, Guy JR, Morcom J (2002) Participation in screening for colorectal cancer based on a faecal occult blood test is improved by endorsement by the primary care practitioner. J Med Screen 9: $147-152$.

Cummings P (2009) Methods for estimating adjusted risk ratios. Stata J 9: 175-196.

Douglas E, Waller J, Duffy SW, Wardle J (2016) Socioeconomic inequalities in breast and cervical screening coverage in England: are we closing the gap? J Med Screen 23: 98-103.

Duffy SW, Myles JP, Maroni R, Mohammad A (2016) Rapid review of evaluation of interventions to improve participation in cancer screening services. J Med Screen. (epub ahead of print, 17 October 2016; doi:10.1177/ 0969141316664757).

Eaker S, Adami HO, Granath F, Wilander E, Sparen P (2004) A large, population-based randomized controlled trial to increase attendance at screening for cervical cancer. Cancer Epidemiol Biomarkers Prev 13: 346-354.

Ferlay J, Soerjomataram I, Ervi M, Dikshit R, Eser S, Mathers C, Rebelo M, Parkin DM, Forman D, Bray F (2013) Cancer Incidence and Mortality Worldwide: IARC Cancer Base No. 11 (Internet). GLOBOCAN 2012 v1.0 Lyon. International Agency for Research on Cancer: France.

Hall NJ, Rubin GP, Dobson C, Weller D, Wardle J, Ritchie M, Rees CJ (2015) Attitudes and beliefs of non-participants in a population-based screening programme for colorectal cancer. Health Expect 18: 1645-1657.

Hardcastle JD, Chamberlain JO, Robinson MH, Moss SM, Amar SS, Balfour TW, James PD, Mangham CM (1996) Randomised controlled trial of faecaloccult-blood screening for colorectal cancer. Lancet 348: 1472-1477.

Hewitson P, Ward AM, Heneghan C, Halloran SP, Mant D (2011) Primary care endorsement letter and a patient leaflet to improve participation in colorectal cancer screening: results of a factorial randomised trial. $\mathrm{Br} \mathrm{J}$ Cancer 105: 475-480.

Kewenter J, Brevinge H, Engaras B, Haglind E, Ahren C (1994) Results of screening, rescreening, and follow-up in a prospective randomized study for detection of colorectal cancer by fecal occult blood testing. Results for 68,308 subjects. Scand J Gastroenterol 29: 468-473.

Kronborg O, Fenger C, Olsen J, Jorgensen OD, Sondergaard O (1996)

Randomised study of screening for colorectal cancer with faecal-occultblood test. Lancet 348: 1467-1471.

Mandel JS, Bond JH, Church TR, Snover DC, Bradley GM, Schuman LM, Ederer F (1993) Reducing mortality from colorectal cancer by screening for fecal occult blood. Minnesota Colon Cancer Control Study. N Engl J Med 328: 1365-1371.

Massat NJ, Khagram LA, Shankleman J, Ariyanayagam S, Garner A, Rainbow S, Duffy SW (2014) An intervention service in a UK Asian community to promote participation in the NHS Bowel Cancer Screening Progamme: results from the pilot study. World J Epidemiol Cancer Prev 3: 31-40.

Raine R, Duffy SW, Wardle J, Solmi F, Morris S, Howe R, Kralj-Hans I, Snowball J, Counsell N, Moss S, Hackshaw A, von Wagner C, Vart G, McGregor LM, Smith SG, Halloran S, Handley G, Logan RF, Rainbow S, Smith S, Thomas MC, Atkin W (2016) Impact of general practice endorsement on the social gradient in uptake in bowel cancer screening. Br J Cancer 114: 321-326.

Senore C, Armaroli P, Silvani M, Andreoni B, Bisanti L, Marai L, Castiglione G, Grazzini G, Taddei S, Gasperoni S, Giuliani O, Malfitana G, Marutti A, Genta G, Segnan N (2010) Comparing different strategies for colorectal cancer screening in Italy: predictors of patients' participation. Am J Gastroenterol 105: 188-198.

Shankleman J, Massat NJ, Khagram L, Ariyanayagam S, Garner A, Khatoon S, Rainbow S, Rangrez S, Colorado Z, Hu W, Parmar D, Duffy SW (2014) Evaluation of a service intervention to improve awareness and uptake of bowel cancer screening in ethnically-diverse areas. Br J Cancer 111: 1440-1447.

StataCorp (2013) Stata Statistical Software: Release. 13. StataCorp LP: College Station, TX, USA.

von Wagner C, Baio G, Raine R, Snowball J, Morris S, Atkin W, Obichere A, Handley G, Logan RF, Rainbow S, Smith S, Halloran S, Wardle J (2011) Inequalities in participation in an organized national colorectal cancer screening programme: results from the first 2.6 million invitations in England. Int J Epidemiol 40: 712-718.

Wardle J, von Wagner C, Kralj-Hans I, Halloran SP, Smith SG, McGregor LM, Vart G, Howe R, Snowball J, Handley G, Logan RF, Rainbow S, Smith S, Thomas MC, Counsell N, Morris S, Duffy SW, Hackshaw A, Moss S, Atkin W, Raine R (2015) Effects of evidence-based strategies to reduce the socioeconomic gradient of uptake in the English NHS Bowel Cancer Screening Programme (ASCEND): four cluster-randomised controlled trials. Lancet 387: 751-759.

Zajac IT, Whibley AH, Cole SR, Byrne D, Guy J, Morcom J, Young GP (2010) Endorsement by the primary care practitioner consistently improves participation in screening for colorectal cancer: a longitudinal analysis. J Med Screen 17: 19-24.

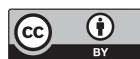

This work is licensed under the Creative Commons Attribution 4.0 International License. To view a copy of this license, visit http://creativecommons.org/licenses/by/4.0/

(C) The Author(s) named above 2017

Supplementary Information accompanies this paper on British Journal of Cancer website (http://www.nature.com/bjc) 